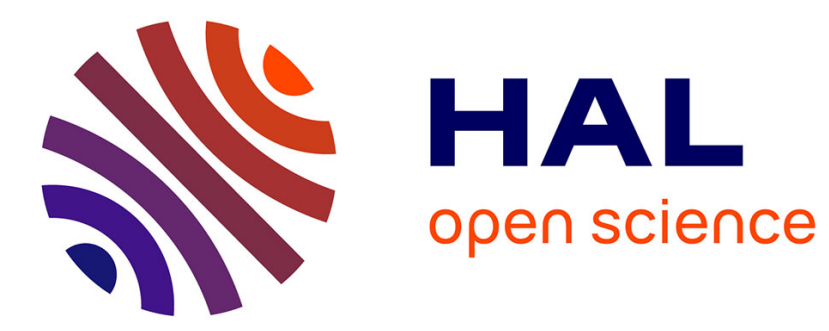

\title{
Running an HCI Experiment in Multiple Parallel Universes
}

Pierre Dragicevic, Fanny Chevalier, Stéphane Huot

\section{To cite this version:}

Pierre Dragicevic, Fanny Chevalier, Stéphane Huot. Running an HCI Experiment in Multiple Parallel Universes. 2014, pp.607-618. 10.1145/2559206.2578881 . hal-00976507

\section{HAL Id: hal-00976507 https://hal.inria.fr/hal-00976507}

Submitted on 9 Apr 2014

HAL is a multi-disciplinary open access archive for the deposit and dissemination of scientific research documents, whether they are published or not. The documents may come from teaching and research institutions in France or abroad, or from public or private research centers.
L'archive ouverte pluridisciplinaire $\mathbf{H A L}$, est destinée au dépôt et à la diffusion de documents scientifiques de niveau recherche, publiés ou non, émanant des établissements d'enseignement et de recherche français ou étrangers, des laboratoires publics ou privés. 


\section{Running an $\mathrm{HCl}$ Experiment in Multiple Parallel Universes}

\begin{tabular}{|c|c|}
\hline Pierre Dragicevic & Pierre Dragicevic \\
\hline $\begin{array}{l}\text { Inria } \\
\text { Pierre Dragicevic }\end{array}$ & $\begin{array}{l}\text { Inria } \\
\text { Pierre Dragicevic }\end{array}$ \\
\hline $\begin{array}{l}\text { Inria } \\
\text { Inte }\end{array}$ & $\begin{array}{l}\text { Inria } \\
\text { Intic }\end{array}$ \\
\hline $\begin{array}{l}\text { Pierre Dragicevic } \\
\text { Inria }\end{array}$ & $\begin{array}{l}\text { Pierre Dragicevic } \\
\text { Inria }\end{array}$ \\
\hline $\begin{array}{l}\text { Pierre Dragicevic } \\
\text { Inria }\end{array}$ & $\begin{array}{l}\text { Pierre Dragicevic } \\
\text { Inria }\end{array}$ \\
\hline $\begin{array}{l}\text { Fanny Chevalier } \\
\text { Inria }\end{array}$ & $\begin{array}{l}\text { Fanny Chevalier } \\
\text { Inria }\end{array}$ \\
\hline $\begin{array}{l}\text { Fanny Chevalier } \\
\text { Inria }\end{array}$ & $\begin{array}{l}\text { Fanny Chevalier } \\
\text { Inria }\end{array}$ \\
\hline $\begin{array}{l}\text { Fanny Chevalier } \\
\text { Inria }\end{array}$ & $\begin{array}{l}\text { Fanny Chevalier } \\
\text { Inria }\end{array}$ \\
\hline $\begin{array}{l}\text { Fanny Chevalier } \\
\text { Inria }\end{array}$ & $\begin{array}{l}\text { Fanny Chevalier } \\
\text { Inria }\end{array}$ \\
\hline $\begin{array}{l}\text { Stéphane Huot } \\
\text { Univ. Paris Sud, CNRS, Inria }\end{array}$ & $\begin{array}{l}\text { Stéphane Huot } \\
\text { Univ. Paris Sud, CNRS, Inria }\end{array}$ \\
\hline $\begin{array}{l}\text { Stéphane Huot } \\
\text { Univ. Paris Sud, CNRS, Inria }\end{array}$ & $\begin{array}{l}\text { Stéphane Huot } \\
\text { Univ. Paris Sud, CNRS, Inria }\end{array}$ \\
\hline $\begin{array}{l}\text { Stéphane Huot } \\
\text { Univ. Paris Sud, CNRS, Inria }\end{array}$ & $\begin{array}{l}\text { Stéphane Huot } \\
\text { Univ. Paris Sud, CNRS, Inria }\end{array}$ \\
\hline $\begin{array}{l}\text { Stéphane Huot } \\
\text { Univ. Paris Sud, CNRS, Inria }\end{array}$ & $\begin{array}{l}\text { Stéphane Huot } \\
\text { Univ, Paris Sud, CNRS, Inria }\end{array}$ \\
\hline \multicolumn{2}{|c|}{$\begin{array}{l}\text { P. Dragicevic, P. Dragicevic, P. Dragicevic, P. Dragicevic, } \\
\text { F. Chevalier, F. Chevalier, F. Chevalier, F. Chevalier, } \\
\text { S. Huot, S. Huot, S. Huot, S. Huot. } \\
\text { Running an HCI Experiment in Multiple Parallel Universes. } \\
\text { i (CHI '14) : Extended Abstracts of the 32nd International Conference } \\
\text { on Human Factors in Computing Systems, ACM, April } 2014 . \\
\text { Authors Version }\end{array}$} \\
\hline
\end{tabular}

\begin{abstract}
We experimentally evaluated a haptic touch slider in 8 parallel universes. The results were overall similar but exhibited surprisingly high variability in terms of statistical significance patterns. We discuss the general implications of these findings for empirical $\mathrm{HCl}$ research.
\end{abstract}

\section{Author Keywords}

Evaluation, Replication, Multiverse, NHST, $p$-value

\section{ACM Classification Keywords}

H.5.2 [User Interfaces]: Evaluation/methodology.

\section{Introduction}

Scientific knowledge in $\mathrm{HCl}$ largely builds on empirical studies. But in a world where time, funding and access to participants are limited, researchers are often left with running studies only once, on a few subjects. Fortunately, the existence of a multiverse [1] allows to parallelize research efforts and alleviate these practical constraints.

A multiverse experiment was conducted to assess the benefits of haptic feedback on touch sliders. Each experiment was conducted and analyzed separately in a different parallel universe, using the same methods and by the "same" investigators. We first provide the eight independent reports, then propose a general discussion. 


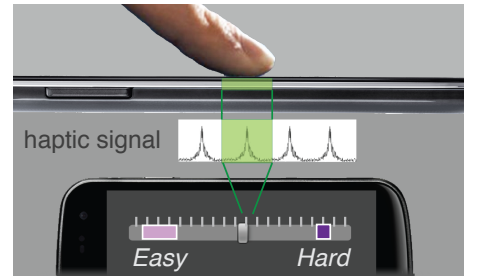

Figure 1: Example of Easy (left) and Hard (right) targets on the touch slider. In the haptic condition (HS), 20 evenly-spaced detents were simulated with vibrotactile feedback.

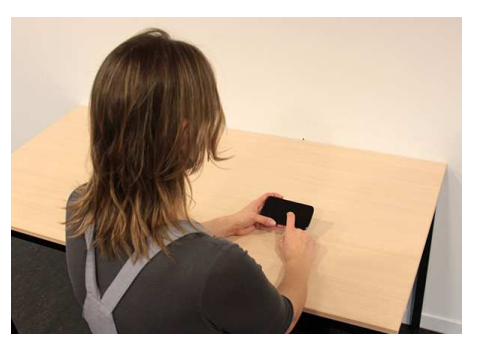

Figure 2: A participant completing our study.

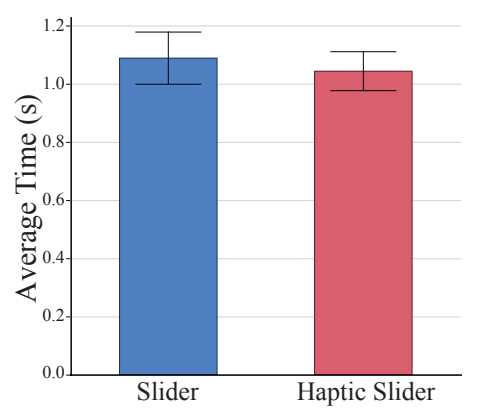

Figure 3: Time by TeChNiQue.

\section{User Study}

Our study examines the benefits of adding haptic detents to touch sliders. We used 1-D target acquisition tasks involving both easy and hard targets (see Figure 1).

A repeated measure full-factorial within-subject design was used. The factors were TECHNIQUE $=\{S=$ slider, $H S=$ haptic slider $\}$, and Difficulty $=\{$ Easy, Hard $\}$. Twelve volunteers (2 female) familiar with touch devices, aged 22-36, participated in the study. We collected a total of 12 PARticipant $\times 2$ TeChNique $\times 2$ Difficulty $\times 128$ repetitions $=6144$ trials with completion TIME.

\section{Hypotheses}

(H1) Technique $H S$ is faster than technique $S$ overall.

(H2) Easy tasks are faster than Hard tasks overall.

\section{Results}

An Anova on Time with the model Technique $\times$ DIFFICULTY $\times$ RND $($ PARTICIPANT $)$ reveals a highly significant effect of TECHNIQUE but no significant effect of Difficulty and no TECHNIQUE $\times$ DifFICULTY interaction (see Table 1).

Table 1: AnOva table.

\begin{tabular}{|l|l|l|l|}
\hline Source & $\mathrm{df}$ & $\mathrm{F}$ & Sig. \\
\hline Technique & 1,11 & 12.7336 & $0.0044^{* *}$ \\
Difficulty & 1,11 & 2.7084 & 0.1281 \\
Technique $\times$ Difficulty & 1,11 & 4.0402 & 0.0696 \\
\hline
\end{tabular}

Our ANOva analysis therefore confirms that technique $H S$ yields significantly shorter completion times than technique $S$ overall, i.e., all task difficulties confounded. The average Time is $1.09 \mathrm{~s}$ for $S$, and $1.04 \mathrm{~s}$ for $H S$ (see Figure 3). This difference corresponds to a $4.8 \%$ increase in speed for technique $H S$ compared to technique $S$.

\section{Discussion}

Our user study shows that subjects completed the tasks significantly faster in the presence of haptic feedback ( $4.8 \%$ faster). Our hypothesis $(\mathrm{H} 1)$ is therefore confirmed.

The superiority of haptic feedback seems to hold for all target difficulties, as suggested by the lack of significant interaction between Technique and Difficulty. Even though large targets do not suffer from the "fat finger" problem, multimodal feedback still seems superior to visual-only feedback. This could be explained by the fact that the haptic channel is a sensory modality directly connected with kinesthetic and motor functions, and therefore capitalizes on our reflexive motor responses.

Surprisingly, we found no significant effect of DifFICULTY overall, so our hypothesis ( $\mathrm{H} 2)$ is not confirmed. This could be explained by the fact that differences in target difficulty were not large enough to significantly affect performance. We could have used different target sizes, but the limited input resolution of the device prevented us from using much smaller targets. Conversely, a very large target would occupy most of the slider range, which does not capture realistic slider tasks. Overall, it seems that for sliders, target size is not a crucial factor.

To summarize, our study provides strong evidence for the benefits of tactile feedback when operating sliders. Although moderate, the effect of technique was found to be highly significant. Tactile guidance provides additional proprioceptive cues when interacting with the glass surface of the device-otherwise uniformly flat. This allows users to maintain an accurate mental model of the slider thumb's location, speeding up the reaching of specific locations. Overall, based on our results, we recommend the use of sliders with haptic detents on touch devices, both for fine and for coarse control. 


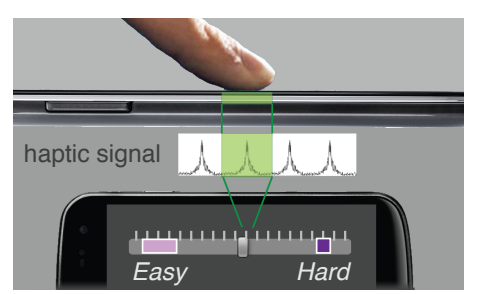

Figure 1: Example of Easy (left) and Hard (right) targets on the touch slider. In the haptic condition (HS), 20 evenly-spaced detents were simulated with vibrotactile feedback.

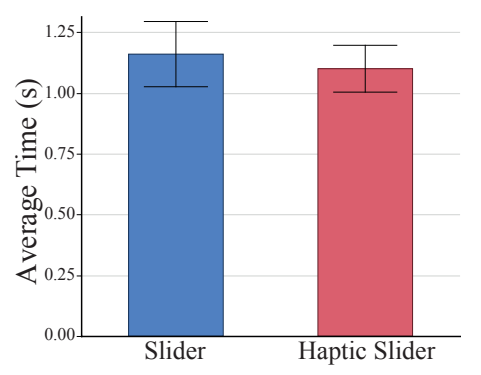

Figure 2: TIME by TeCHNIQUe.

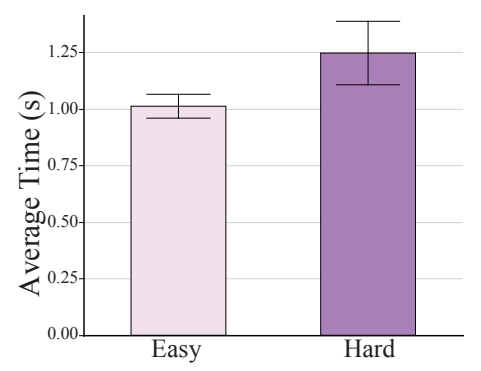

Figure 3: Time by Difficulty.

\section{User Study}

Our study examines the benefits of adding haptic detents to touch sliders. We used 1-D target acquisition tasks involving both easy and hard targets (see Figure 1).

A repeated measure full-factorial within-subject design was used. The factors were TECHNIQUE $=\{S=$ slider, $H S=$ haptic slider $\}$, and Difficulty $=\{$ Easy, Hard $\}$. Twelve volunteers ( 3 female) familiar with touch devices, aged 20-37, participated in the study. We collected a total of 12 PARTICIPANT $\times 2$ TEChNiQUe $\times 2$ Difficulty $\times 128$ repetitions $=6144$ trials with completion Time.

\section{Hypotheses}

(H1) Technique $H S$ is faster than technique $S$ overall.

(H2) Easy tasks are faster than Hard tasks overall.

\section{Results}

An Anova on Time with the model Technique $\times$

DifFICULTY $\times$ RND $($ PARTICIPANT) reveals a significant effect of both TECHNiQue and DifficulTy, but no significant TECHNIQUE $\times$ DiffiCUlTy interaction effect (see Table 1).

Table 1: ANOva table.
\begin{tabular}{|l|l|l|l|}
\hline Source & $\mathrm{df}$ & $\mathrm{F}$ & Sig. \\
\hline Technique & 1,11 & 5.1139 & $0.0450^{*}$ \\
Difficulty & 1,11 & 6.2892 & $0.0291^{*}$ \\
TechniquexDifficulty & 1,11 & 1.3669 & 0.2671 \\
\hline
\end{tabular}

Our analysis therefore confirms that $H S$ is faster than $S$ overall, with an average Time of 1.16 s for $S$ vs. 1.10 s for $H S$, a $5.5 \%$ increase in speed (see Figure 2). Our analysis also confirms the effects of task difficulty, with an average TIME of $1.25 \mathrm{~s}$ for Hard vs. 1.01s for Easy,

corresponding to a $23.8 \%$ increase in speed (see Figure 3 ).

\section{Discussion}

Our user study shows that subjects completed the tasks significantly faster in the presence of haptic feedback (5.5\% faster). Our hypothesis $(\mathrm{H} 1)$ is therefore confirmed.

The superiority of haptic feedback seems to hold for all target difficulties, as suggested by the lack of significant interaction between TECHNIQUE and Difficulty. Even though large targets do not suffer from the "fat finger" problem, multimodal feedback still seems superior to visual-only feedback. This could be explained by the fact that the haptic channel is a sensory modality directly connected with kinesthetic and motor functions, and therefore capitalizes on our reflexive motor responses.

Our analysis also shows a significant difference between the two levels of difficulty all techniques confounded, with Easy being as much as $23.8 \%$ faster than Hard.

Therefore, our hypothesis $(\mathrm{H} 2)$ is also supported. We derived our difficulty levels based on extensive pilot studies, so as not to favor any technique. Our results validate our experimental design and confirm that target size is an adequate metric for task difficulty. $H S$ appears to perform comparably well under two widely different task difficulties, suggesting that its advantages may well generalize to other difficulty levels.

To summarize, our study confirms that adding tactile feedback in the form of simulated detents facilitates the operation of sliders. Tactile guidance provides additional proprioceptive cues when interacting with the glass surface of the device-otherwise uniformly flat. This likely allows users to maintain an accurate mental model of the slider thumb's location, speeding up the reaching of specific locations. Overall, based on our results, we recommend the use of sliders with haptic detents on touch devices, both for fine and for coarse control. 


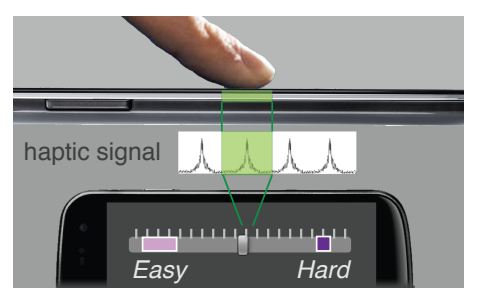

Figure 1: Example of Easy (left) and Hard (right) targets on the touch slider. In the haptic condition (HS), 20 evenly-spaced detents were simulated with vibrotactile feedback.

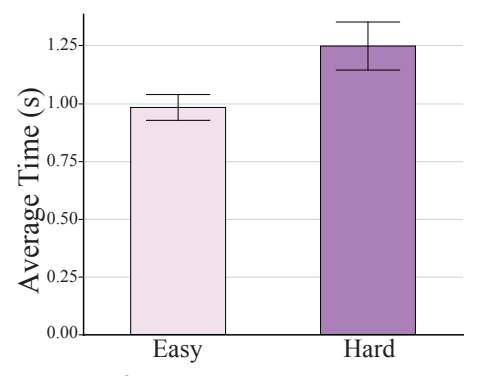

Figure 2: Time by Difficulty.

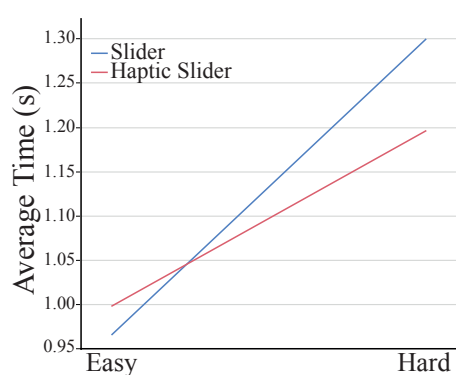

Figure 3: Time by Difficulty and TeChnique.

\section{User Study}

Our study examines the benefits of adding haptic detents to touch sliders. We used 1-D target acquisition tasks involving both easy and hard targets (see Figure 1).

A repeated measure full-factorial within-subject design was used. The factors were TECHNIQUE $=\{S=$ slider, $H S=$ haptic slider $\}$, and Difficulty $=\{$ Easy, Hard $\}$. Twelve volunteers ( 4 female) familiar with touch devices, aged 18-32, participated in the study. We collected a total of 12 PARticipant $\times 2$ TeChNique $\times 2$ Difficulty $\times 128$ repetitions $=6144$ trials with completion Time.

\section{Hypotheses}

(H1) Technique $H S$ is faster than technique $S$.

(H2) Easy tasks are faster than Hard.

\section{Results}

An Anova on Time with the model Technique $\times$

DifFICULTY $\times$ RND (PARTICIPANT) reveals no significant effect of TECHNique, but a highly significant effect of DifFICULTY with also a highly significant

TeChNique $\times$ Difficulty interaction effect (see Table 1 ).

Table 1: ANova table.
\begin{tabular}{|l|l|l|l|}
\hline Source & $\mathrm{df}$ & $\mathrm{F}$ & Sig. \\
\hline Technique & 1,11 & 3.2748 & 0.0977 \\
Difficulty & 1,11 & 14.2324 & $0.0031^{* *}$ \\
TechniquexDifficulty & 1,11 & 14.9541 & $0.0026^{* *}$ \\
\hline
\end{tabular}

Our analysis confirms the effect of difficulty (avg. Times: Easy $=0.98 \mathrm{~s}$, Hard $=1.25 \mathrm{~s}$, see Figure 2). Student's t-tests reveal no significant difference between techniques for

Easy (avg. Times: $S=0.96 \mathrm{~s}, H S=1.00 \mathrm{~s}, p=0.1416$ ), and a highly significant difference between techniques for Hard, with a $9.2 \%$ increase in speed with HS (avg. Times: $S=1.30 \mathrm{~s}, H S=1.19 \mathrm{~s}, p=0.0069)$ (see Figure 3 ).

\section{Discussion}

While we did not observe a significant main effect of Technique, an analysis of simple effects reveals that HS significantly outperformed $S$ in the Hard condition, with as much as $9.2 \%$ in speed improvement. Therefore, our hypothesis $(\mathrm{H} 1)$ is only partially confirmed.

Although we did not find a significant difference between techniques in the Easy condition, Figure 3 exhibits an intriguing trend, raising the possibility of $H S$ being worse than $S$ under the Easy condition. This seems to be confirmed by the very strong interaction observed between TeChnique and Difficulty. A possible explanation could be that the regular bursts generated by the haptic detents is distracting to some users, which in turn slightly impairs their performance. Indeed, some participants expressed discomfort while interacting with $H S$.

In the Hard condition, however, the situation is very different: due to the "fat finger" problem, users are likely deprived of visual cues during the corrective phase of their movement. In this case, multimodal feedback likely alleviates this issue by providing non-visual guidance. In other terms, when the target is small, the benefits brought by haptic feedback largely outweigh discomfort issues, allowing users to acquire these targets much more easily.

To summarize, our study shows that adding tactile feedback in the form of simulated detents can be an effective solution to the "fat finger" problem when manipulating sliders on touch devices. However, haptic feedback can also be distracting and in some cases, impair performance when the task is easy (large 1-D targets). Overall, based on our results, we recommend the use haptic detents on touch sliders for tasks that require fine control, but not for tasks where coarse control is sufficient. 


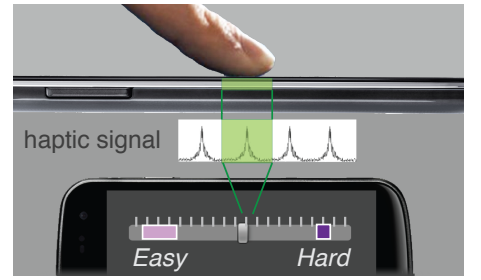

Figure 1: Example of Easy (left) and Hard (right) targets on the touch slider. In the haptic condition (HS), 20 evenly-spaced detents were simulated with vibrotactile feedback.

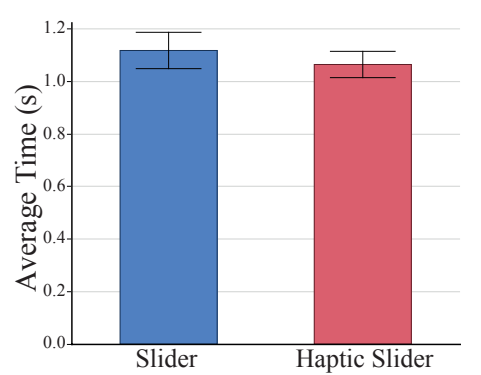

Figure 2: TIME by TECHNIQUE.

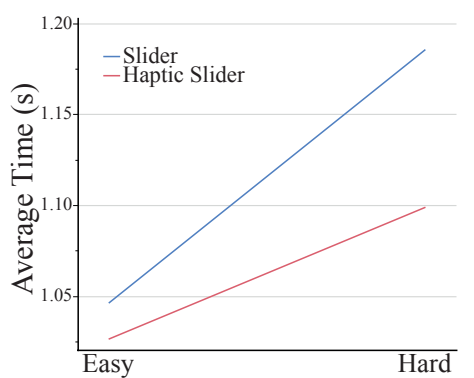

Figure 3: Time by Difficulty and TECHNIQUe.

\section{User Study}

Our study examines the benefits of adding haptic detents to touch sliders. We used 1-D target acquisition tasks involving both easy and hard targets (see Figure 1).

A repeated measure full-factorial within-subject design was used. The factors were TECHNIQUE $=\{S=$ slider, $H S=$ haptic slider $\}$, and Difficulty $=\{$ Easy, Hard $\}$. Twelve volunteers ( 5 female) familiar with touch devices, aged $21-50$, participated in the study. We collected a total of 12 PARTICIPANT $\times 2$ TEChNiQUe $\times 2$ Difficulty $\times 128$ repetitions $=6144$ trials with completion Time.

\section{Hypotheses}

(H1) Technique $H S$ is faster than technique $S$ overall.

(H2) Easy tasks are faster than Hard tasks overall.

\section{Results}

An Anova on Time with the model TeChniquex

DifFICULTY $\times$ RND (PARTICIPANT) reveals a significant effect of TECHNIQUE and a significant interaction Technique $\times$ Difficulty (see Table 1 ).

Table 1: Anova table.
\begin{tabular}{|l|l|l|l|}
\hline Source & $\mathrm{df}$ & $\mathrm{F}$ & Sig. \\
\hline Technique & 1,11 & 7.2144 & $0.0212^{*}$ \\
Difficulty & 1,11 & 4.1479 & 0.0665 \\
TechniquexDifficulty & 1,11 & 5.5941 & $0.0375^{*}$ \\
\hline
\end{tabular}

Our analysis therefore confirms that $H S$ is faster than $S$ overall, with an average Time of $1.12 \mathrm{~s}$ for $S$ vs. $1.06 \mathrm{~s}$ for $H S$, a $5.7 \%$ increase in speed (see Figure 2). Student's t-tests reveal no significant difference between techniques for Easy (avg. Times: $S=1.05 \mathrm{~s}, H S=1.03 \mathrm{~s}, p=0.4065$ ), and a highly significant difference between techniques for Hard, with a $8.2 \%$ increase in speed with $H S$ (avg. Times: $S=1.19 \mathrm{~s}, H S=1.10 \mathrm{~s}, p=0.0060$ ) (see Figure 3 ).

\section{Discussion}

Our user study shows that subjects completed the tasks significantly faster in the presence of haptic feedback (5.7\% faster). Our hypothesis $(\mathrm{H} 1)$ is therefore confirmed.

In addition, we found a significant interaction between technique and task difficulty, with a higher performance gain brought by HS for the Hard condition (8.2\% faster). In contrast, the improvement was lower $(1.9 \%)$ under the Easy condition (also see Figure 3). One explanation is that in the Hard condition, the "fat finger" problem interferes with the corrective phase of users' movement. Multimodal feedback likely alleviates this by providing non-visual guidance. Under the Easy condition, the target was larger and the fat finger issue not as pronounced, making haptic feedback still useful but less critical.

Surprisingly, we were not able to find a significant effect of Difficulty overall, despite the trends visible in Figure 3. This could be explained by the fact that differences in the target difficulty were not large enough to significantly affect performance. In our pilot studies we considered tasks involving much smaller or much larger targets, but dismissed them as unrealistic. So it seems that overall, target size is not a crucial factor for sliders.

To summarize, our study confirms that adding tactile feedback in the form of simulated detents facilitates the operation of sliders. Tactile guidance provides additional proprioceptive cues when interacting with the glass surface of the device-otherwise uniformly flat. Operating sliders is hard on touch devices in general, but even more so when fine control is needed, due to the "fat finger" problem. We show that haptic guidance greatly facilitates this task. Overall, based on our results, we recommend the use of sliders with haptic detents on touch devices, especially when fine control is needed. 


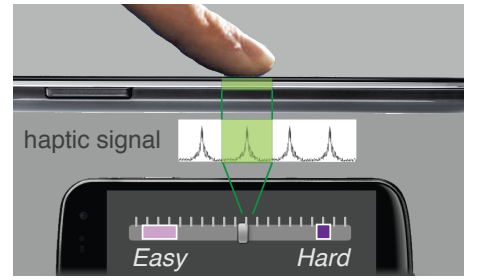

Figure 1: Example of Easy (left) and Hard (right) targets on the touch slider. In the haptic condition (HS), 20 evenly-spaced detents were simulated with vibrotactile feedback.

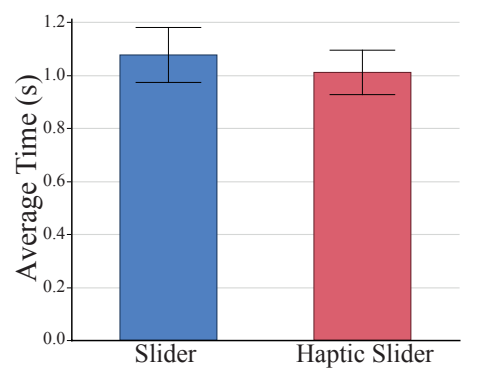

Figure 2: TIME by TECHNIQUE.

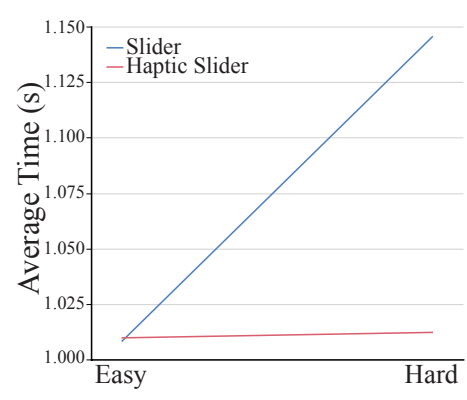

Figure 3: Time by Difficulty and TEChNique.

\section{User Study}

Our study examines the benefits of adding haptic detents to touch sliders. We used 1-D target acquisition tasks involving both easy and hard targets (see Figure 1).

A repeated measure full-factorial within-subject design was used. The factors were TECHNIQUE $=\{S=$ slider, $H S=$ haptic slider $\}$, and Difficulty $=\{$ Easy, Hard $\}$. Twelve volunteers ( 4 female) familiar with touch devices, aged 18-39, participated in the study. We collected a total of 12 PARticipant $\times 2$ TeChNique $\times 2$ Difficulty $\times 128$ repetitions $=6144$ trials with completion Time.

\section{Hypotheses}

(H1) Technique $H S$ is faster than technique $S$ overall.

(H2) Easy tasks are faster than Hard tasks overall.

\section{Results}

An Anova on Time with the model Technique $\times$ DifFICULTY $\times$ RND (PARTICIPANT) reveals a significant effect of TECHNIQUE and a significant interaction Technique $\times$ Difficulty (see Table 1 ).

Table 1: Anova table.
\begin{tabular}{|l|l|l|l|}
\hline Source & df & F & Sig. \\
\hline Technique & 1,11 & 6.0536 & $0.0317^{*}$ \\
Difficulty & 1,11 & 1.0392 & 0.3299 \\
Techniquex Difficulty & 1,11 & 9.4480 & $0.0106^{*}$ \\
\hline
\end{tabular}

Our analysis therefore confirms that $H S$ is faster than $S$ overall, with an average Time of $1.08 \mathrm{~s}$ for $S$ vs. $1.01 \mathrm{~s}$ for $H S$, a $6.9 \%$ increase in speed (see Figure 2). Student's t-tests reveal no significant difference between techniques for Easy (avg. Times: $S=1.01 \mathrm{~s}, H S=1.01 \mathrm{~s}, p=0.9601$ ), and a highly significant difference between techniques for Hard, with a $12.9 \%$ increase in speed with HS (avg. Times: $S=1.14 \mathrm{~s}, H S=1.01 \mathrm{~s}, p=0.0071$ ) (see Figure 3 ).

\section{Discussion}

Our user study shows that subjects completed the tasks significantly faster in the presence of haptic feedback $(6.9 \%$ faster $)$. Our hypothesis $(\mathrm{H} 1)$ is therefore confirmed.

In addition, we found a significant interaction between technique and task difficulty, with a higher performance gain brought by $H S$ for the Hard condition (as much as $12.9 \%$ faster). In contrast, the two techniques seem to perform very similarly under the Easy condition (see Figure 3). One explanation is that in the Hard condition, users are deprived of visual cues during the corrective phase of their movement because of the "fat finger" problem. Multimodal feedback likely alleviates this by providing non-visual guidance. Under the Easy condition, the target may have been large enough for users to rely on visual feedback only, making haptic feedback superfluous.

Surprisingly, we were not able to find a significant effect of Difficulty overall. A tentative explanation can be found in Figure 3: while $S$ seems to be affected by difficulty, $H S$ exhibits a stable performance across difficulty levels. This suggests that with haptic feedback, all targets are equally easy. Although this seems to contradict Fitts' Law, recall this law is about aimed movements with visual feedback.

The haptic channel may not be as sensitive to target size, possibly due to the fact that it is a sensory modality directly connected with kinesthetic and motor functions.

To summarize, our study shows that adding tactile feedback in the form of simulated detents facilitates the precise manipulation of sliders. Precise control of sliders is challenging on touch devices, partly due to the "fat finger" problem. We show that with haptic guidance, it becomes practically as easy as coarse control. Overall, based on our results, we recommend the use of sliders with haptic detents on touch devices when fine control is needed. 


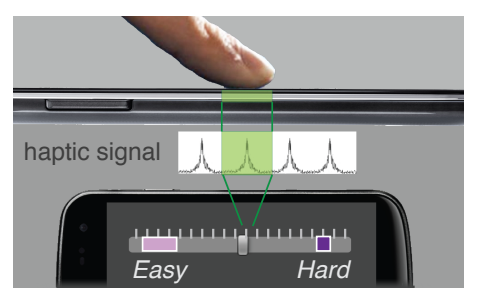

Figure 1: Example of Easy (left) and Hard (right) targets on the touch slider. In the haptic condition (HS), 20 evenly-spaced detents were simulated with vibrotactile feedback.

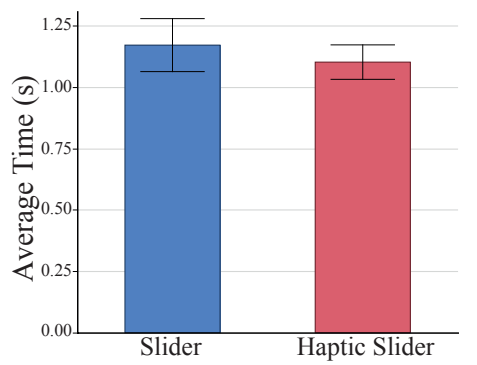

Figure 2: TIME by TECHNIQUE.

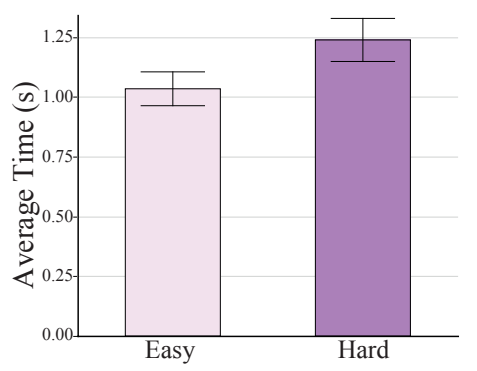

Figure 3: Time by Difficulty and TECHNIQUE.

\section{User Study}

Our study examines the benefits of adding haptic detents to touch sliders. We used 1-D target acquisition tasks involving both easy and hard targets (see Figure 1).

A repeated measure full-factorial within-subject design was used. The factors were TECHNIQUE $=\{S=$ slider, $H S=$ haptic slider $\}$, and Difficulty $=\{$ Easy, Hard $\}$. Twelve volunteers (2 female) familiar with touch devices, aged $20-43$, participated in the study. We collected a total of 12 PARTICIPANT $\times 2$ TEChNiQUe $\times 2$ Difficulty $\times 128$ repetitions $=6144$ trials with completion Time.

\section{Hypotheses}

(H1) Technique $H S$ is faster than technique $S$ overall.

(H2) Easy tasks are faster than Hard tasks overall.

\section{Results}

An Anova on Time with the model Technique $\times$ DifFICULTY $\times$ RND (PARTICIPANT) reveals a highly significant effect of TECHNIQUE, and a very highly significant effect of Difficulty, and no

TEChNique $\times$ Difficulty interaction (see Table 1 ).

Table 1: Anova table.
\begin{tabular}{|l|l|l|l|}
\hline Source & $\mathrm{df}$ & $\mathrm{F}$ & Sig. \\
\hline Technique & 1,11 & 13.1323 & $0.0040^{* *}$ \\
Difficulty & 1,11 & 21.9758 & $0.0007^{* * *}$ \\
Technique $\times$ Difficulty & 1,11 & 3.9159 & 0.0734 \\
\hline
\end{tabular}

Our analysis therefore confirms that $H S$ is faster than $S$ overall, with an average Time of $1.17 \mathrm{~s}$ for $S$ vs. $1.10 \mathrm{~s}$ for $H S$, a $6.4 \%$ increase in speed (see Figure 2). Our analysis also confirms the effects of task difficulty, with an average TIME of $1.24 \mathrm{~s}$ for Hard vs. 1.03s for Easy,

corresponding to a $20.4 \%$ increase in speed (see Figure 3 ).

\section{Discussion}

Our user study shows that subjects completed the tasks significantly faster in the presence of haptic feedback (6.4\% faster). Our hypothesis ( $\mathrm{H} 1)$ is therefore confirmed.

The superiority of haptic feedback seems to hold for all target difficulties, as suggested by the lack of significant interaction between Technique and Difficulty. Even though large targets do not suffer from the "fat finger" problem, multimodal feedback still seems superior to visual-only feedback. This could be explained by the fact that the haptic channel is a sensory modality directly connected with kinesthetic and motor functions, and therefore capitalizes on our reflexive motor responses.

Our analysis also shows a highly significant difference between the two levels of difficulty all techniques confounded, with Easy being as much as $20.4 \%$ faster than Hard. Therefore, our hypothesis (H2) is also supported. We derived our difficulty levels based on extensive pilot studies, so as not to favor any technique. Our results validate our experimental design and confirm that target size is an adequate metric for task difficulty. $H S$ appears to perform comparably well under two widely different task difficulties, suggesting that its advantages may well generalize to other difficulty levels.

To summarize, our study confirms that adding tactile feedback in the form of simulated detents facilitates the operation of sliders. Tactile guidance provides additional proprioceptive cues when interacting with the glass surface of the device-otherwise uniformly flat. This likely allows users to maintain an accurate mental model of the slider thumb's location, speeding up the reaching of specific locations. Overall, based on our results, we recommend the use of sliders with haptic detents on touch devices, both for fine and for coarse control. 


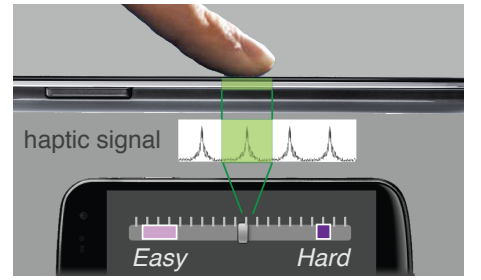

Figure 1: Example of Easy (left) and Hard (right) targets on the touch slider. In the haptic condition (HS), 20 evenly-spaced detents were simulated with vibrotactile feedback.

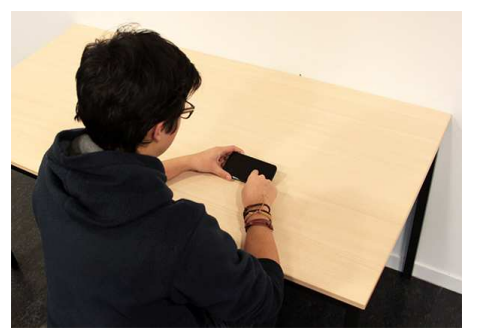

Figure 2: A participant completing our study.

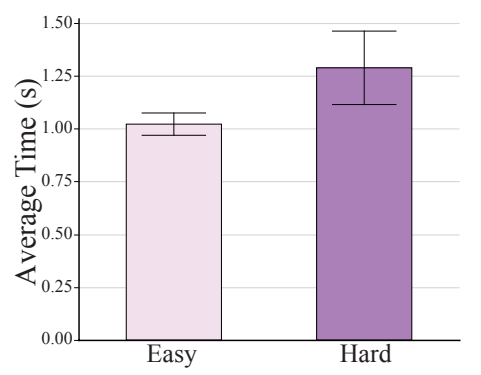

Figure 3: Time by Difficulty and TECHNIQUE.

\section{User Study}

Our study examines the benefits of adding haptic detents to touch sliders. We used 1-D target acquisition tasks involving both easy and hard targets (see Figure 1).

A repeated measure full-factorial within-subject design was used. The factors were TECHNIQUE $=\{S=$ slider, $H S=$ haptic slider $\}$, and Difficulty $=\{$ Easy, Hard $\}$. Twelve volunteers ( 7 female) familiar with touch devices, aged 19-31, participated in the study. We collected a total of 12 PARticipant $\times 2$ TeChNique $\times 2$ Difficulty $\times 128$ repetitions $=6144$ trials with completion TIME.

\section{Hypotheses}

(H1) Technique $H S$ is faster than technique $S$ overall.

(H2) Easy tasks are faster than Hard tasks overall.

\section{Results}

An Anova on Time with the model Technique $\times$

DifFICULTY $\times$ RND $($ PARTICIPANT) reveals no significant effect of TeChnique, but a significant effect of

Difficulty. Furthermore, the ANOvA analysis did not reveal any significant TECHNIQUE $\times$ DIFFICULTY interaction effect (see Table 1 below).

Table 1: ANOvA table.
\begin{tabular}{|l|l|l|l|}
\hline Source & df & F & Sig. \\
\hline Technique & 1,11 & 4.6215 & 0.0547 \\
Difficulty & 1,11 & 4.8698 & $0.0495^{*}$ \\
Technique $\times$ Difficulty & 1,11 & 1.8322 & 0.2030 \\
\hline
\end{tabular}

Our analysis confirms the effects of task difficulty, with an average Time of 1.29 s for Hard vs. $1.02 \mathrm{~s}$ for Easy, corresponding to a $26.5 \%$ increase in speed (see Figure 3 ). Thus our second hypothesis $(\mathrm{H} 2)$ is confirmed.
Discussion

Our initial hypothesis was that haptic feedback would facilitate 1-D target acquisition tasks (H1). Our analyses failed to support this hypothesis. Yet, our results suggest that if haptic feedback may not help, it does not harm either. Indeed, $H S$ was still on average $4 \%$ faster than $S$, although this difference was not statistically significant.

Participants' answers to our post-experiment questionnaire suggest that haptic feedback may provide qualitative benefits beyond pure task completion times. Many participants rated the technique high in hedonistic value (a median of 4 on a 5-point Likert scale), and feedback on haptic detents was overall positive.

The feedback collected during our study also helped us identify directions for improvement for our current prototype. Some participants expressed discomfort while interacting with $H S$. One mentioned "a feeling similar as if the device was sending little electrical shocks to the finger", and thought the equipment was dysfunctional. We believe this could easily be fixed by allowing users to personalize the haptic signal. One participant commented that haptic feedback "feels weird. [She] would rather expect [her] finger to smoothly glide on the glass surface". Indeed, a flat screen provides conflicting affordances with haptic feedback. Visual techniques that emphasize physicality (e.g. shadow or cushion effects to convey holes and bumps) could address this problem.

In summary, while our study did not reveal significant quantitative benefits of haptic detents over the traditional touch slider, the qualitative feedback we received was very positive and encouraging. We were able to collect valuable insights that shed light on the limitations of current haptic interfaces. We hope that our results will inform and inspire further development in the area. 


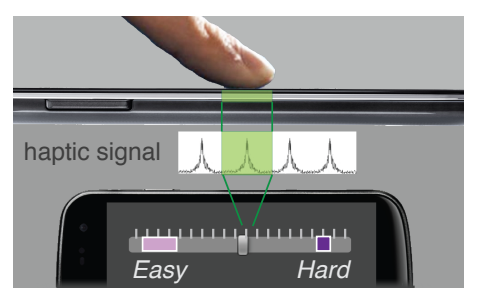

Figure 1: Example of Easy (left) and Hard (right) targets on the touch slider. In the haptic condition (HS), 20 evenly-spaced detents were simulated with vibrotactile feedback.

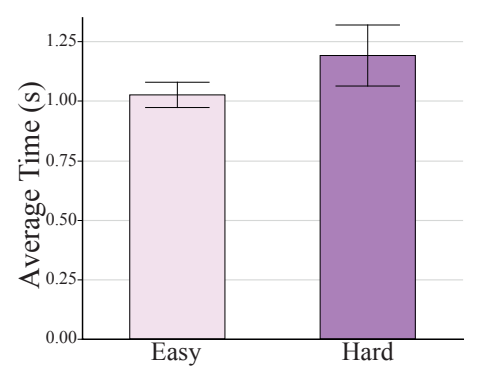

Figure 2: TIme by TeChNiQue.

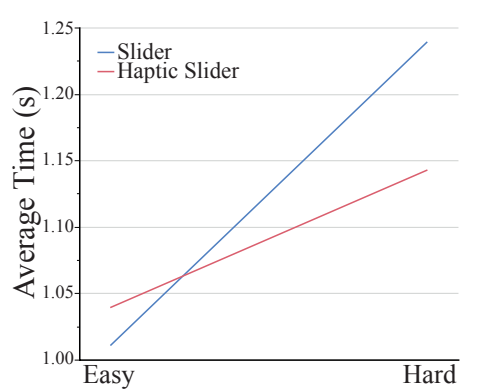

Figure 3: Time by Difficulty and TECHNIQUe.

\section{User Study}

Our study examines the benefits of adding haptic detents to touch sliders. We used 1-D target acquisition tasks involving both easy and hard targets (see Figure 1).

A repeated measure full-factorial within-subject design was used. The factors were TECHNIQUE $=\{S=$ slider, $H S=$ haptic slider $\}$, and Difficulty $=\{$ Easy, Hard $\}$. Twelve volunteers ( 5 female) familiar with touch devices, aged 19-35, participated in the study. We collected a total of 12 PARTICIPANT $\times 2$ TEChNiQUe $\times 2$ Difficulty $\times 128$ repetitions $=6144$ trials with completion Time.

\section{Hypotheses}

$(\mathrm{H} 1)$ Technique $H S$ is faster than technique $S$.

(H2) Easy tasks are faster than Hard.

\section{Results}

An Anova on Time with the model TeChniquex

DifFICULTY $\times$ RND $($ PARTICIPANT) reveals no significant effect of TECHNiQUE, but a significant effect of

DifFICULTY with also a very highly significant

TECHNIQUE $\times$ Difficulty interaction effect (see Table1).

Table 1: ANOvA table.
\begin{tabular}{|l|l|l|l|}
\hline Source & $\mathrm{df}$ & $\mathrm{F}$ & Sig. \\
\hline Technique & 1,11 & 2.1350 & 0.1719 \\
Difficulty & 1,11 & 5.1621 & $0.0442^{*}$ \\
Technique $\times$ Difficulty & 1,11 & 22.6791 & $0.0006^{* * *}$ \\
\hline
\end{tabular}

Our analysis confirms the effect of difficulty (avg. Times: Easy $=1.02 \mathrm{~s}$, Hard $=1.19 \mathrm{~s}$, see Figure 2). Student's t-tests reveal no significant difference between techniques for

Easy (avg. Times: $S=1.01 \mathrm{~s}, H S=1.04 \mathrm{~s}, p=0.2757$ ), and a very highly significant difference between techniques for Hard, with a $8.8 \%$ increase in speed with HS (avg.

Times: $S=1.24 \mathrm{~s}, H S=1.14 \mathrm{~s}, p=0.0061$ ) (see Figure 3 ).

\section{Discussion}

While we did not observe a significant main effect of Technique, an analysis of simple effects reveals that $H S$ significantly outperformed $S$ in the Hard condition, with as much as $8.8 \%$ in speed improvement. Therefore, our hypothesis $(\mathrm{H} 1)$ is only partially confirmed.

Although we did not find a significant difference between techniques in the Easy condition, Figure 3 exhibits an intriguing trend, raising the possibility of $H S$ being worse than $S$ under the Easy condition. This seems to be confirmed by the very strong interaction observed between TeChnique and Difficulty. A possible explanation could be that the regular bursts generated by the haptic detents is distracting to some users, which in turn slightly impairs their performance. Indeed, some participants expressed discomfort while interacting with $H S$.

In the Hard condition, however, the situation is very different: due to the "fat finger" problem, users are likely deprived of visual cues during the corrective phase of their movement. In this case, multimodal feedback likely alleviates this issue by providing non-visual guidance. In other terms, when the target is small, the benefits brought by haptic feedback largely outweigh discomfort issues, allowing users to acquire these targets much more easily.

To summarize, our study shows that adding tactile feedback in the form of simulated detents can be an effective solution to the "fat finger" problem when manipulating sliders on touch devices. However, haptic feedback can also be distracting and in some cases, impair performance when the task is easy (large 1-D targets). Overall, based on our results, we recommend the use haptic detents on touch sliders for tasks that require fine control, but not for tasks where coarse control is sufficient. 

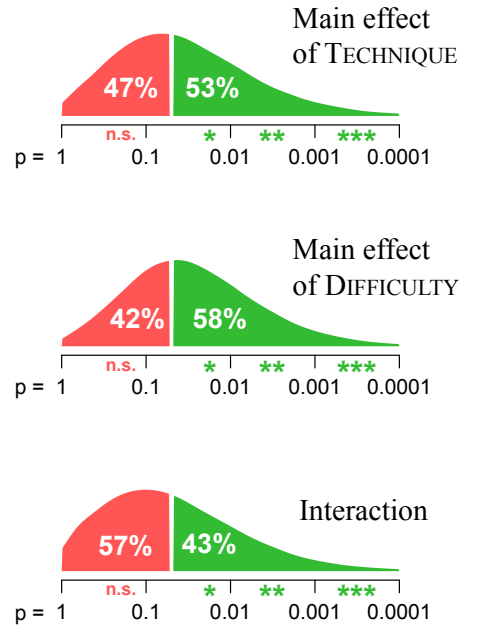

Effect of

TECHNIQUE
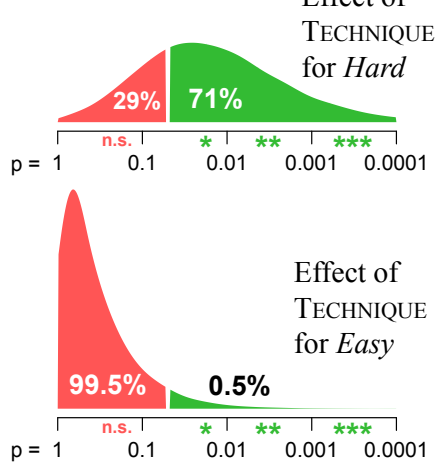

Figure 4: Probability distributions for $p$ values estimated using Monte Carlo methods. Red indicates non-significant, green indicates significant. Except for the null effect (bottom), about any $p$ value can be obtained.

\section{Methods and Discussion}

Setting up a multiverse experiment is impractical today, due to the current difficulty of communicating across universes [1]. We therefore simulated the data that could have been produced by such an experiment. We assumed 8 universes sharing identical characteristics in terms of the population of interest, the true effects, the investigating researchers, the experimental protocol and the data analysis methods. Only population sampling was assumed to be subject to random variations, i.e., the 12 subjects who signed up for the study differed across universes.

A mean Time measure was generated for all 48 combinations of (subject, TEChNIQUe and Difficulty) as follows: $\operatorname{Time}(i, H S, E a s y)=e^{x_{i}}$,

$\operatorname{Time}(i, S, E$ Easy $)=e^{x_{i}+x_{i}^{\prime}}, \operatorname{Time}(i, H S, H a r d)=e^{x_{i}+z_{i}}$, $\operatorname{Time}(i, S, H a r d)=e^{x_{i}+y_{i}+z_{i}}$ with $X, X^{\prime} \sim \mathcal{N}(0,0.1)$, $Y \sim \mathcal{N}(0.08,0.1), Z \sim \mathcal{N}(0.1,0.2) . \mathcal{N}\left(\mu, \sigma^{2}\right)$ denotes a normal distribution and $x_{i}$ refers to the realization of the random variable $X$ for the subject $i$. This method yields lognormal time distributions and correlated measures within subjects. Values of $\mu$ and $\sigma^{2}$ have been chosen to yield statistical powers of 0.4 to 0.7 (see Figure 4 ). The two techniques have identical means for Easy.

This exercise is meant to illustrate the extent to which experiment analyses and conclusions are determined by chance. Our analysis methods are typical of $\mathrm{HCl}$, with statistical powers typical of psychology [3] and $\mathrm{HCl}$ [4].

Researchers know about sampling error but are overly obsessed with Type I errors (which did not occur in any of our 8 universes). Our analyses highlight a more general and widespread pitfall: the overreliance on $p$ values. If $p$ is small, means are reported and discussed as if they were exact. A large $p$ value (i.e., larger than the standard but nonetheless arbitrary cutoff of 0.05 ) is often taken as a sign that there is no effect. But $p$ values simply cannot be trusted (see Figure 4, and [2] for a demo). Although traditional statistical practices have started to be questioned in $\mathrm{CHI}$ [4], this issue has been disregarded. We refer the reader to [3] for a more extensive discussion and an alternative: relying on estimation rather than $p$ values when analyzing and interpreting experimental results.

Note that our simulated multiverse experiment is equivalent to simulating multiple replications of an experiment in a single universe [3]. There are indeed a number of analogies: like the multiverse theory, the principle of scientific replication has theoretical support but has been hardly observed in practice. In the context of $\mathrm{HCl}$, we thought that a multiverse scenario would be slightly more believable [5]. It also captures the idea that while many outcomes are possible for an experiment, we typically only have access to one of them. Hopefully, we will always keep the multiverse in mind.

\section{References}

[1] Carr, B., Ed. Universe or multiverse? Cambridge University Press, 2007.

[2] Cumming, G. Dance of the $\mathrm{p}$ values (video). tinyurl.com/danceptrial2, 2009.

[3] Cumming, G. The new statistics why and how. Psychological science 25, 1 (2014), 7-29.

[4] Kaptein, M., and Robertson, J. Rethinking statistical analysis methods for CHI. In Proc. CHI' 12, ACM (2012), 1105-1114.

[5] Wilson, M. L., Mackay, W., Chi, E., Bernstein, M., Russell, D., and Thimbleby, H. RepliCHI - CHI should be replicating and validating results more: discuss. In CHI Extended abstracts, ACM (2011), 463-466. 